\title{
Incomplete revascularization: what the surgeon needs to know
}

\author{
Dror B. Leviner, Gianluca Torregrossa, John D. Puskas \\ Department of Cardiovascular Surgery, Icahn School of Medicine at Mount Sinai, New York, NY, USA \\ Correspondence to: John D. Puskas, MD, FACS, FACC. Professor of Cardiothoracic Surgery, Icahn School of Medicine at Mount Sinai, Mount Sinai \\ Heart at Saint Luke's, 1111 Amsterdam Avenue, New York, NY 10025, USA. Email: John.puskas@mountsinai.org.
}

\begin{abstract}
For many years, the concept of "complete revascularization" (CR) was considered an absolute truth in coronary surgery with improved long-term survival and a lower rate of reintervention. This was derived from early publications which showed a survival benefit for patients undergoing coronary artery bypass grafting (CABG) who received CR. Many advances in the field of coronary revascularization have been made in the years that passed since those publications, including more frequent use of percutaneous coronary intervention (PCI) in patients with multivessel disease (MVD). This has led some to question the importance of CR and raise the option of "reasonable incomplete revascularization" (IR) for selected patients. The definition of $\mathrm{CR}$ is variable in the literature with the two most common definitions being an anatomical (revascularization of all coronary segments with stenosis and larger than a predefined size) and a functional definition (where revascularization is considered complete if all ischemic and viable territories are reperfused). No randomized control trials have been conducted to compare complete versus IR, and a significant proportion of data is based on post hoc analysis of data from randomized control trials and registries. Multiple studies have proven that $\mathrm{CR}$ is achieved more frequently with CABG then with PCI. A review of the available data from the past three to four decades shows a trend toward improved results with CR, regardless of the reperfusion strategy chosen. This should impact the heart team discussion when choosing a revascularization strategy and impact the surgical decision making while preforming CABG. IR can be part of a hybrid revascularization strategy or be reserved for rare cases where the cost of achieving CR much outweighs the benefit.
\end{abstract}

Keywords: Coronary artery bypass grafting (CABG); off pump coronary artery bypass grafting (OPCAB); incomplete revascularization (IR); percutaneous coronary intervention (PCI); hybrid coronary revascularization (HCR)

Submitted Apr 13, 2018. Accepted for publication May 24, 2018.

doi: 10.21037/acs.2018.06.07

View this article at: http://dx.doi.org/10.21037/acs.2018.06.07

\section{Introduction}

For many years, the concept of "complete revascularization" (CR) was considered an absolute truth in coronary surgery. This was based on early publications showing a survival benefit for patients receiving CR $(1,2)$. However, much has changed in the field of coronary revascularization since these seminal papers. The patient population referred to surgery is older and suffers from more co-morbidities. Surgical technique has also changed substantially with more frequent use of arterial grafts, off-pump coronary artery bypass surgery (OPCAB), and improved perioperative management. Secondary prevention strategies have become more aggressive and effective for patients with ischemic heart disease. And perhaps most importantly, percutaneous coronary interventions (PCI) have evolved rapidly to become a true alternative for the treatment of patients with multi-vessel coronary disease, along with the option of hybrid coronary revascularization (HCR). The current ESC/EACTS guidelines on myocardial revascularization state that $\mathrm{CR}$ is recommended (class I, level of evidence B) whereas the ACCF/AHA guidelines do not give any direct recommendation regarding $\mathrm{CR}(3,4)$.

Multiple publications have addressed the issue of incomplete revascularization (IR) in past decades. Some have reinforced the notion that CR is an important goal in 
coronary artery bypass grafting (CABG), whereas others have questioned this paradigm. Most of these publications are from registries or post hoc analyses but it is unlikely that a randomized trial of CR vs. IR will ever be held. This review will attempt to answer one of the questions that has been part of coronary surgery from its early days: is CR the "only option" in coronary surgery or is there room for "reasonable" IR as part of a "tailored therapy" approach in a world of sicker patients and multiple treatment strategies.

\section{Definition of $\mathbf{C R}$}

One of the major obstacles to reaching a uniform conclusion regarding the importance of CR is the lack of a uniform definition of CR. In general, CR can by defined by either an anatomical definition or a functional definition. The anatomic classification can be a broad one, implying that all stenotic vessels should be revascularized, regardless of size or territory supplied. A more common anatomic definition is that all vessels with a stenosis $>50 \%$ and a size of $>1.5 \mathrm{~mm}$ are revascularized (5). A functional definition, on the other hand, refers to revascularization only of coronary arteries that supply viable myocardium. Revascularization can therefore be functionally complete, but not anatomically complete. Most trials regarding CR in coronary surgery used the anatomic classification since viability data was not available. Moreover, the definition relies on the operative report since postoperative angiography is rarely used. This differs to PCI trials, where completion angiography can be examined. It must also be emphasized that failure after initial CR (acute thrombosis with PCI, acute graft failure in CABG) will not count directly as IR, but rather will affect the clinical outcome.

In an attempt to find the most appropriate definition of IR, different definitions of CR were retrospectively applied to the BARI surgical arm (6). The four definitions of CR used in BARI were-definition 1: "traditional" CR was defined as all diseased arterial systems (stenosis $\geq 50 \%$ ) receiving at least one graft insertion ( $83 \%$ of study population). Definition 2: "functional" CR was defined as bypassing all diseased "primary" (according to a coronary artery map defined by the investigators) coronary segments (74\% of study population). Definition 3: patients were grouped by whether the number of distal anastomoses was less than (30\% of study population), equal to (34\%) or greater than $(36 \%)$ the number of diseased coronary segments. Definition 4: patients were grouped by whether they had $\geq 2$ distal site insertions to both the left anterior descending coronary artery (LAD) and to a non-LAD arterial system ( $8 \%$ of study population), $\geq 2$ distal sites to the LAD (28\%), $\geq 2$ distal site insertions to a non-LAD arterial system (14\%) or by whether no arterial system had multiple distal site insertions (50\%). The results of their 7-year follow-up of 1,484 patients showed no independent survival advantage for traditional or functional CR as compared with IR. For the fourth definition, seven-year death/myocardial infarction (MI) was highest (32.9\%) when more than one anastomosis was constructed to any non-LAD system. No increased risk was associated with constructing more than one anastomosis into the LAD system.

\section{IR in on pump CABG}

The first trial to systematically address completeness of revascularization was the coronary artery surgery study (CASS). The study analyzed the outcomes of 3,372 patients with 3 -vessel disease who underwent isolated first-time CABG between July 1974 and June 1979 with a mean follow-up of 4.9 years (7). The completeness of revascularization was defined by the number of the three major arteries that received bypass anastomoses. Only $16 \%$ of patients in the trial received an internal thoracic artery graft, while the average number of bypasses was 3.2. Patients with more CR ( 3 or more vessel bypass vs. 1 or 2 vessel bypass) had improved survival [relative risk (RR) of mortality $0.75,95 \%$ confidence interval (CI): 0.59-0.94, $\mathrm{P}=0.01)$ and event-free survival (RR of mortality and combined cardiac events of death, MI, reoperation, or definite angina $0.87,95 \%$ CI: $0.78-0.96, \mathrm{P}=0.01$ ) and were more likely to have less severe angina or be asymptomatic. Patients with ejection fraction $<35 \%$ (according to a subset analysis) who had three or more vessels bypassed had improved survival compared to those who had two vessel bypasses.

Scott et al. reported on their registry of 2,067 patients who underwent an isolated left internal thoracic artery (LITA) graft to the LAD between 1971 and 1997. Of these, $26 \%$ had 2 vessel disease and $13 \%$ had 3 -vessel disease (8). The most common reason for IR was small vessel size. Of interest, the prevalence of IR fell from about $60 \%$ in the first years of the series to $20-25 \%$ by the early 1980 s and remained at those levels thereafter. Compared with a $75 \% 20$-year survival in patients with no non-left anterior descending disease, those with either left circumflex or left main trunk disease experienced a $44 \%$ survival, and 
those with proximal right coronary artery disease, $42 \%$. The authors concluded that the LITA-LAD graft, by itself, does not fully compensate for the presence of disease in other coronary systems.

The BARI trial, as mentioned above, enrolled patients from August 1988 to 1991 (6). The authors found that no independent advantage was conferred by CR, defined by either the traditional or functional definitions, although the risk estimates were in the direction that favored CR.

The multicenter Arterial Revascularization Therapies Study (ARTS) trial, conducted between 1997 and 1998, enrolled 1,205 patients with multivessel disease (MVD) who could be potentially completely and equivalently revascularized with either PCI or CABG (9). CR was achieved in $84.1 \%$ of the surgically treated patients and $70.5 \%$ of the angioplasty patients $(\mathrm{P}<0.001)$, based on an anatomic definition. After one year of follow up, there was no significant difference in event-free survival between surgically treated patients with CR and those with IR (87.8\% in the incomplete group vs. $89.9 \%$ in the complete group), but patients randomized to stenting with IR had a greater need for subsequent bypass surgery and thus a lower rate of event free survival. This was reinforced when long term outcomes were published and showed no significant difference in 5-year survival without major adverse cardiac and cerebrovascular events (MACCEs; death, cerebrovascular accident, $\mathrm{MI}$, and any revascularization) between patients with CR and IR treated with PCI and between patients with CR and IR treated with CABG (10).

In a single-center retrospective analysis of 1,034 patients who underwent first-time CABG with a mean followup of 3.3 years, the authors chose what they defined as a functional classification of IR (with CR defined as the placement of at least 1 bypass graft distal to a $>50 \%$ narrowing in each diseased territory) (11). This cohort had a mean age of 68 years and included mostly on pump CABG (80.4\% of cases). The most common reasons recorded for incomplete surgical revascularization were that the arteries were too small, that they were severely diseased, or both. In this study, IR was associated with a 5-year unadjusted increased overall mortality rate $(47.4 \%$ versus $17.6 \%$, respectively, $\mathrm{P}<0.001)$ and cardiac mortality rate $(25.5 \%$ versus $6.9 \%, \mathrm{P}<0.001)$. After adjustment for predictors of death, IR remained an independent risk factor for death [hazard ratio (HR), 1.85, 95\% CI: 1.03-3.34, $\mathrm{P}=0.04$ for allcause death, and HR, 1.73, 95\% CI: $1.18-2.55, \mathrm{P}=0.006$ for cardiac death only].

Rastan et al. (12) reported on 936 consecutive patients (that represent $10.6 \%$ of their total registry) undergoing "reasonable" IR (all of which had a LITA to the LAD, with 2.44 distal anastomosis on average, compared to 2.97 in the CR group). On-pump CABG had been used in $80 \%$ of the patients, with notably longer average bypass time and cross clamp time for the IR group. They found no difference in hospital mortality (3.3\% CR versus $3.2 \% \mathrm{IR}$ ) or in cumulative survival at 1 year (93.1\% CR versus $93.6 \%$ IR) and 5 years (82.2 CR versus $80.9 \%$ IR).

A large series by Kim et al. (13) studied 1,914 consecutive Asian patients with multivessel coronary disease undergoing drug-eluting stents (DES) implantation $(n=1,400)$ or CABG $(n=514$, about $25 \%$ OPCAB). In the CABG group, the rate of CR was $66.9 \%$. Over a 5 -year follow-up, the authors did not find a significant difference in outcomes between CR and IR for both percutaneous and surgical revascularization, except in those patients with multivessel IR ( $\geq 2$ diseased vessels incompletely revascularized); among these was a trend in favor of CR for the composite end point of death, MI, stroke, and repeat revascularization (30.3\% versus $22.1 \%)$. Indeed, this apparent lack of statistically significant results for the comparison of CR versus IR may relate fundamentally to inadequate sample size in many of the studies described above.

The SYNTAX trial patients were also used to assess whether IR by PCI or CABG influences long-term outcomes (14). IR was defined to have occurred when a preoperatively identified vessel (as agreed upon by a surgeon and an interventional cardiologist) with a lesion was not revascularized. IR was found in $36.8 \%$ of CABG patients. Among CABG patients, no difference in MACCE at 3 years was seen between the IR and CR groups. Independent predictors of IR by CABG were unstable angina, diffuse disease or narrowed ( $<2 \mathrm{~mm}$ ) segment distal to the lesion and the number of diseased vessels. A post hoc analysis of the 4-year results of the same trial were later published (15). Of note, this analysis included both the randomized and the nested registries. Here, CR was achieved in $66.9 \%$ of all CABG patients. Within the PCI and CABG arms, IR (compared with CR) was associated with significantly higher frequencies of 4-year mortality, all-cause revascularization, stent thrombosis (PCI arm), and MACCE. The authors comment on the discrepancy between these results and those of the aforementioned trial and conclude that it probably reflects the fact that the nested CABG registry contains more anatomically complex patients compared with the randomized CABG cohort (as evidenced by higher SYNTAX scores in the nested group). 
The Second Medicine, Angioplasty, or Surgery Study (MASS II), was a randomized trial comparing treatment for patients with stable multivessel coronary artery disease and preserved systolic ventricular function (16). In the CABG group, $92 \%$ of the patients received at least one LITA, with $3.3 \pm 0.8$ anastomosis per patient. Of these patients, $72.2 \%$ had CR according to an anatomic definition (significantly more than in the PCI group). In an analysis of the ten-year outcomes there was no difference in long term freedom from cardiovascular mortality between patients who received CR versus IR in the CABG group but significantly higher cardiovascular mortality in patients who underwent PCI with IR compared with CR.

A meta-analysis of studies comparing CR to IR included 35 studies from 1970 to 2012 with 89,883 patients (17). Of the studies included, 28 were observational studies, 5 were subgroup analysis of randomized controlled trials (RCTs), 1 was a subgroup analysis of a non-RCT, and 1 was a singlecenter RCT comparing CR to IR. The authors concluded that $\mathrm{CR}$ was more often achieved with CABG than with $\mathrm{PCI}$ and is associated with a $30 \%$ reduction in long-term mortality, a $22 \%$ reduction in $\mathrm{MI}$, and a $26 \%$ reduction in repeat coronary revascularization procedures. The lower mortality associated with CR was seen in both PCI- and CABG-treated patients and was independent of the study design and definition of CR. This is arguably the most statistically powerful and rigorous assessment in the peerreviewed literature of the impact of CR and IR among both PCI a CABG patients with multivessel CAD.

Kieser and colleagues explored the hypothesis that total arterial revascularization balances survival between CR and IR (18). They examined 1,000 consecutive patients, $60 \%$ of which were operated on with OPCAB between 2003 and 2012. CR was defined by an anatomic definition. These patients received $98 \%$ arterial grafts and $85 \%$ had CR. Operative mortality was $3.8 \%$ overall, $8.6 \%$ for patients with IR, and $3.2 \%$ for patients with $\mathrm{CR}(\mathrm{P}=0.008)$. For operative mortality using multivariable logistic regression, after controlling for European System for Cardiac Operative Risk Evaluation category $(\mathrm{P}<0.001)$ and $\mathrm{CABG}$ urgency $(\mathrm{P}=0.03)$, there was no evidence of a statistically significant increased risk of death due to IR. For midterm follow-up (after controlling for European System for Cardiac Operative Risk Evaluation category $(\mathrm{P}<0.001)$ and comorbidities $(\mathrm{P}=0.017))$, there was a significant interaction between age $\geq 80$ years and IR $(\mathrm{P}=0.017)$ in predicting mortality. The adjusted HR associated with IR for patients older than age 80 years was 5.7 versus 1.2 for younger patients. They concluded that the use of arterial conduits, because of the longevity of the grafts, balances survival between CR and IR and mitigates against the disadvantages of IR.

A recent study examined the long-term impact of IR using a single center registry including 8,570 patients who underwent first time CABG from 1995-2007 (19). Requirements for CR involved having each diseased coronary territory receive at least $1 \mathrm{graft}$. CR was achieved in $81 \%$ of the patients, with the right coronary artery most often not grafted, followed by the circumflex. IR rates were higher in patients with the following preoperative characteristics: increasing age, diabetes, renal failure, peripheral vascular disease, cerebral vascular disease, low ejection fraction, and congestive heart failure. After adjusting for clinical differences between the groups, IR was an independent predictor of death (HR, 1.2; 95\% CI: 1.1-1.4; $\mathrm{P}=0.0001)$. IR was also an independent predictor of readmission to hospital for cardiac reasons (HR, 1.2; 95\% CI: $1.0-1.3 ; \mathrm{P}=0.02$ ).

\section{IR in off-pump CABG (OPCAB)}

In the early years of OPCAB there was concern whether it carried an inherent risk of IR. Later publications have refuted that claim and proven that in experienced hands, OPCAB surgery results in a degree of revascularization that is comparable to conventional CABG (20). A number of publications have addressed the subject of IR specifically in OPCAB.

Caputo et al. reviewed 1,479 OPCAB operations (30\% of total CABG volume) done in a single institution from April 1996 to December 2002 (21). Patients with MVD were classified as having IR if the number of diseased coronary systems exceeded the number of distal anastomoses. Patients that had IR (16\% of the patients) tended to be older and were female, had more extensive disease, worse dyspnea, a higher Parsonnet score, poorer ejection fraction, congestive cardiac failure, asthma or chronic obstructive airways disease, and previous cardiac surgery. The adjusted HR for patient survival with IR versus CR was 1.56 (95\% CI: $1.19-2.06 ; \mathrm{P}=0.001$ ) but only during the first 4 to 6 months after surgery. They concluded that the patient's preoperative condition, rather than the IR itself, might play a major role in the adverse outcomes of the IR patients.

Completeness of revascularization was also assessed in a large single-center retrospective review comparing on pump CABG and OPCAB (22). The cohort included more than 
twelve thousand patients who received CABG over a period of 10 years ending in 2006. Patients were divided into four groups-1 to 3 grafts operated on-pump $v s$. off-pump and 4-7 grafts operated on-pump vs. off-pump. Completeness of revascularization was assessed according to the index of completeness of revascularization (ICOR). The ICOR was defined as the number of distal grafts constructed divided by the number of diseased vessels reported on the preoperative coronary arteriogram. The ICOR was significantly lower among patients with 1 to 3 grafts than among patients with 4 to 7 grafts $(\mathrm{P}<0.001)$ and was also significantly lower for patients treated with $\mathrm{OPCAB}$ than for those treated with on-pump CABG $(\mathrm{P}<0.001)$. Proportional hazards regression analysis showed no significant influence of surgery type or number of grafts on long-term (up to 10 years) survival within the four groups. However, higher ICOR was associated with improved long-term survival within all groups.

A recent report evaluated the impact of IR on long term outcomes in 1,553 OPCAB patients of whom $87 \%$ had CR (23). Patients were considered to have received IR when the number of distal anastomoses was less than the number of diseased vessels. The follow-up duration was 60 months. Propensity score-based patient matching was performed to adjust for a lower incidence of chronic obstructive pulmonary disease and peripheral artery occlusive disease among CR patients compared with IR patients. After creating two propensity matched groups of 200 patients each, the CR group showed superior 5-year cardiac survival compared with the IR group $(96.2 \% \pm 1.4 \%$ versus $88.8 \% \pm 2.5 \%, \mathrm{P}=0.022$ ), with a similar freedom from MACCE rate. IR was identified as an independent predictor of cardiac death. IR predicted cardiac death more distinctly in patients with a lower left ventricular ejection fraction (LVEF <35\%).

\section{IR in HCR}

HCR, i.e., the combination of minimally invasive surgical treatment of part of the coronary disease (namely the LAD) and PCI to the other significant lesions is an attractive option for either high risk patients, or a subset of patients with anatomically suitable disease, as it avoids sternotomy and the use of cardiopulmonary bypass. It also inherently means the patient receives an IR, at least for a limited time, unless the two procedures are combined in one session (which is infrequently the case). Although in some hybrid series completeness of revascularization is as high as $100 \%$ (24), in studies with broader inclusion criteria, completeness of revascularization occurred in as few as $68 \%$ of patients $(25)$.

Lichtenberg et al. (26) examined a cohort of 411 patients who underwent minimally invasive direct coronary artery bypass graft (MIDCAB) of the LAD. Of these patients, $63.7 \%$ had isolated disease of the LAD, while the rest had MVD. The latter group received IR for multiple reasons (very small target vessels $(<1.0 \mathrm{~mm}$ diameter), stenosis of less than $50 \%$, distal localization of the stenosis, longterm patency after angioplasty, or an extensive risk for sternotomy and (or) cardiopulmonary bypass). The mean follow-up was 29.4 months. After adjustment of baseline characteristics by Cox regression analysis, the 3-year risk of cardiac death was significantly higher in the MVD group (RR, 2.2, 95\% CI: 1.8-4.65; P=0.029). They concluded that MVD was an independent risk factor for adverse outcome in patients undergoing minimally invasive revascularization of the LAD using the internal thoracic artery, but with acceptable midterm morbidity and mortality.

\section{IR in PCI}

The impact of IR in PCI has been investigated for more than three decades. Within this time frame (as with CABG), interventional techniques have significantly and rapidly improved, including the implementation of bare metal and subsequently DES over balloon angioplasty alone. This has had major implications on the ability to compare studies over time and called into question the present relevance of studies performed before the era of routine DES and current improvements in post-interventional medical therapy, including more advanced antiplatelet therapy and aggressive risk factor reduction.

In broad terms, CR is achieved less frequently with PCI than with CABG. A recent meta-analysis of CR in PCI included 38 trials with more than 150 thousand patients (27). The studies included were mostly observational and included large registries or post hoc analyses of randomized trials (many of them mentioned here, including SYNTAX, BARI, ARTS II, and MASS II), with previous meta-analysis and trials comparing target lesion only revascularization versus upfront multivessel revascularization in non-culprit lesions for ST elevation MI (STEMI) patients excluded. CR was achieved in less than half of patients. Odds of death (OR, 0.69, 95\% CI: 0.61-0.78), repeat revascularization, $\mathrm{MI}$, and major adverse cardiac events were significantly lower in the patients 
who underwent CR. These outcomes were unchanged on subgroup analysis regardless of the definition of CR. Similar findings were obtained when CR was studied in the chronic total occlusion (CTO) subgroup. The authors concluded that these associations between IR and adverse clinical outcomes suggest that in patients with MVD, consideration should be given to the degree of revascularization that can be achieved by PCI when discussing choice of revascularization modality within the heart team, in addition to consideration of lesion complexity, functional significance, patient characteristics, and SYNTAX score, consistent with current international guidelines.

\section{Conclusions}

Careful review of data from the last three to four decades (with the inherent changes in surgical technique, postoperative care, and more aggressive risk factor management after surgery) reveals a rather consistent trend toward survival benefit of CR over IR for patients with MVD undergoing CABG. This is especially true for patients who are older and have worse anginal symptoms or reduced LVEF.

These data on CR should consciously impact decisionmaking at two stages. Firstly (as part of the heart team discussion), the likelihood of achieving CR with different modalities should be considered, since lower CR rates are consistently achieved with PCI and IR is related to worse outcomes. Once it has been decided that the patient will undergo surgical revascularization, all efforts must be made to achieve CR.

The option of "reasonable IR" should be reserved for very unusual cases in which a careful assessment of the risks and benefits of alternative approaches; including culprit lesion PCI, CR by PCI, IR by (minimally invasive) CABG and CR by CABG with arterial conduits; taking into consideration the comorbidities and personal wishes of the individual patient, leads to a multidisciplinary team decision for less than CR.

\section{Acknowledgements}

None.

\section{Footnote}

Conflicts of Interest: The authors have no conflicts of interest to declare.

\section{References}

1. Buda AJ, Macdonald IL, Anderson MJ, et al. Long-term results following coronary bypass operation. Importance of preoperative actors and complete revascularization. J Thorac Cardiovasc Surg 1981;82:383-90.

2. Jones EL, Craver JM, Guyton RA, et al. Importance of complete revascularization in performance of the coronary bypass operation. Am J Cardiol 1983;51:7-12.

3. Authors/Task Force members, Windecker S, Kolh P, et al. 2014 ESC/EACTS Guidelines on myocardial revascularization: The Task Force on Myocardial Revascularization of the European Society of Cardiology (ESC) and the European Association for CardioThoracic Surgery (EACTS)Developed with the special contribution of the European Association of Percutaneous Cardiovascular Interventions (EAPCI). Eur Heart J 2014;35:2541-619.

4. Hillis LD, Smith PK, Anderson JL, et al. 2011 ACCF/ AHA Guideline for Coronary Artery Bypass Graft Surgery: a report of the American College of Cardiology Foundation/American Heart Association Task Force on Practice Guidelines. Circulation 2011;124:e652-735.

5. Bypass Angioplasty Revascularization Investigation I. Comparison of coronary bypass surgery with angioplasty in patients with multivessel disease. $\mathrm{N}$ Engl J Med 1996;335:217-25.

6. Vander Salm TJ, Kip KE, Jones RH, et al. What constitutes optimal surgical revascularization? Journal of the American College of Cardiology 2002;39:565-72.

7. Bell MR, Gersh BJ, Schaff HV, et al. Effect of completeness of revascularization on long-term outcome of patients with three-vessel disease undergoing coronary artery bypass surgery. A report from the Coronary Artery Surgery Study (CASS) Registry. Circulation 1992;86:446-57.

8. Scott R, Blackstone EH, McCarthy PM, et al. Isolated bypass grafting of the left internal thoracic artery to the left anterior descending coronary artery. J Thorac Cardiovasc Surg 2000;120:173-84.

9. van den Brand MJ, Rensing BJ, Morel MA, et al. The effect of completeness of revascularization on event-free survival at one year in the arts trial. J Am Coll Cardiol 2002;39:559-64.

10. Sarno G, Garg S, Onuma Y, et al. Impact of completeness of revascularization on the five-year outcome in percutaneous coronary intervention and coronary artery bypass graft patients (from the ARTS-II study). Am J Cardiol 2010;106:1369-75. 
11. Kleisli T, Cheng W, Jacobs MJ, et al. In the current era, complete revascularization improves survival after coronary artery bypass surgery. J Thorac Cardiovasc Surg 2005;129:1283-91.

12. Rastan AJ, Walther T, Falk V, et al. Does reasonable incomplete surgical revascularization affect early or long-term survival in patients with multivessel coronary artery disease receiving left internal mammary artery bypass to left anterior descending artery? Circulation 2009;120:S70-7.

13. Kim YH, Park DW, Lee JY, et al. Impact of angiographic complete revascularization after drug-eluting stent implantation or coronary artery bypass graft surgery for multivessel coronary artery disease. Circulation 2011;123:2373-81.

14. Head SJ, Mack MJ, Holmes DR Jr, et al. Incidence, predictors and outcomes of incomplete revascularization after percutaneous coronary intervention and coronary artery bypass grafting: a subgroup analysis of 3-year SYNTAX data. Eur J Cardiothorac Surg 2012;41:535-41.

15. Farooq V, Serruys PW, Garcia-Garcia HM, et al. The negative impact of incomplete angiographic revascularization on clinical outcomes and its association with total occlusions: the SYNTAX (Synergy Between Percutaneous Coronary Intervention with Taxus and Cardiac Surgery) trial. J Am Coll Cardiol 2013;61:282-94.

16. Vieira RD, Hueb W, Gersh BJ, et al. Effect of complete revascularization on 10-year survival of patients with stable multivessel coronary artery disease: MASS II trial. Circulation 2012;126:S158-63.

17. Garcia S, Sandoval Y, Roukoz H, et al. Outcomes after complete versus incomplete revascularization of patients with multivessel coronary artery disease: a meta-analysis of 89,883 patients enrolled in randomized clinical trials and observational studies. J Am Coll Cardiol 2013;62:1421-31.

18. Kieser TM, Curran HJ, Rose MS, et al. Arterial grafts balance survival between incomplete and complete revascularization: a series of 1000 consecutive coronary artery bypass graft patients with $98 \%$ arterial grafts. J

Cite this article as: Leviner DB, Torregrossa G, Puskas JD. Incomplete revascularization: what the surgeon needs to know. Ann Cardiothorac Surg 2018;7(4):463-469. doi: 10.21037/ acs.2018.06.07
Thorac Cardiovasc Surg 2014;147:75-83.

19. Mocanu V, Buth KJ, Kelly R, et al. Incomplete revascularization after coronary artery bypass graft operations is independently associated with worse longterm survival. Ann Thorac Surg 2014;98:549-55.

20. Wijeysundera DN, Beattie WS, Djaiani G, et al. Offpump coronary artery surgery for reducing mortality and morbidity: meta-analysis of randomized and observational studies. J Am Coll Cardiol 2005;46:872-82.

21. Caputo M, Reeves BC, Rajkaruna C, et al. Incomplete revascularization during $\mathrm{OPCAB}$ surgery is associated with reduced mid-term event-free survival. Ann Thorac Surg 2005;80:2141-7.

22. Lattouf OM, Thourani VH, Kilgo PD, et al. Influence of on-pump versus off-pump techniques and completeness of revascularization on long-term survival after coronary artery bypass. Ann Thorac Surg 2008;86:797-805.

23. Yi G, Youn YN, Joo HC, et al. Association of incomplete revascularization with long-term survival after off-pump coronary artery bypass grafting. J Surg Res 2013;185:166-73.

24. Cisowski M, Morawski W, Drzewiecki J, et al. Integrated minimally invasive direct coronary artery bypass grafting and angioplasty for coronary artery revascularization. Eur J Cardiothorac Surg 2002;22:261-5.

25. Zenati M, Cohen HA, Griffith BP. Alternative approach to multivessel coronary disease with integrated coronary revascularization. J Thorac Cardiovasc Surg 1999;117:43944; discussion 44-6.

26. Lichtenberg A, Klima U, Paeschke H, et al. Impact of multivessel coronary artery disease on outcome after isolated minimally invasive bypass grafting of the left anterior descending artery. Ann Thorac Surg 2004;78:487-91.

27. Nagaraja V, Ooi SY, Nolan J, et al. Impact of Incomplete Percutaneous Revascularization in Patients With Multivessel Coronary Artery Disease: A Systematic Review and Meta-Analysis. J Am Heart Assoc 2016;5(12). pii: e004598. 